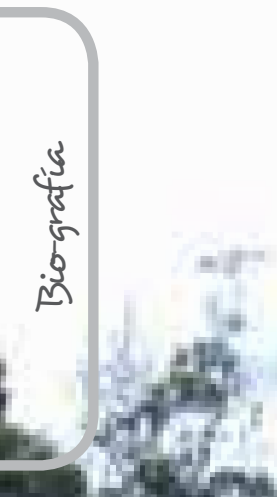

i. $-4+2+1$

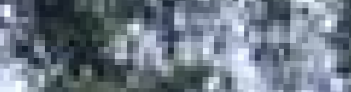

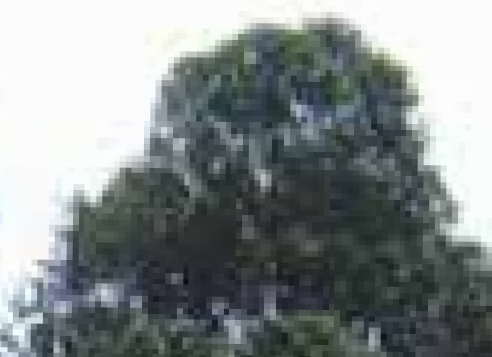

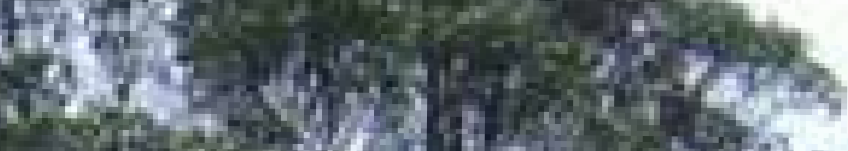

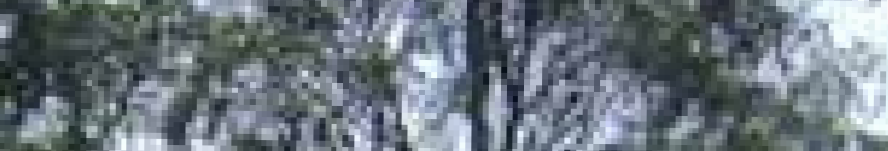
HAf

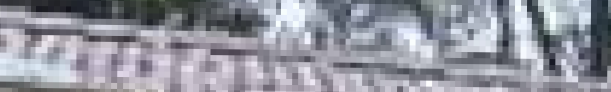
$a y+2 y+2 y^{2}$
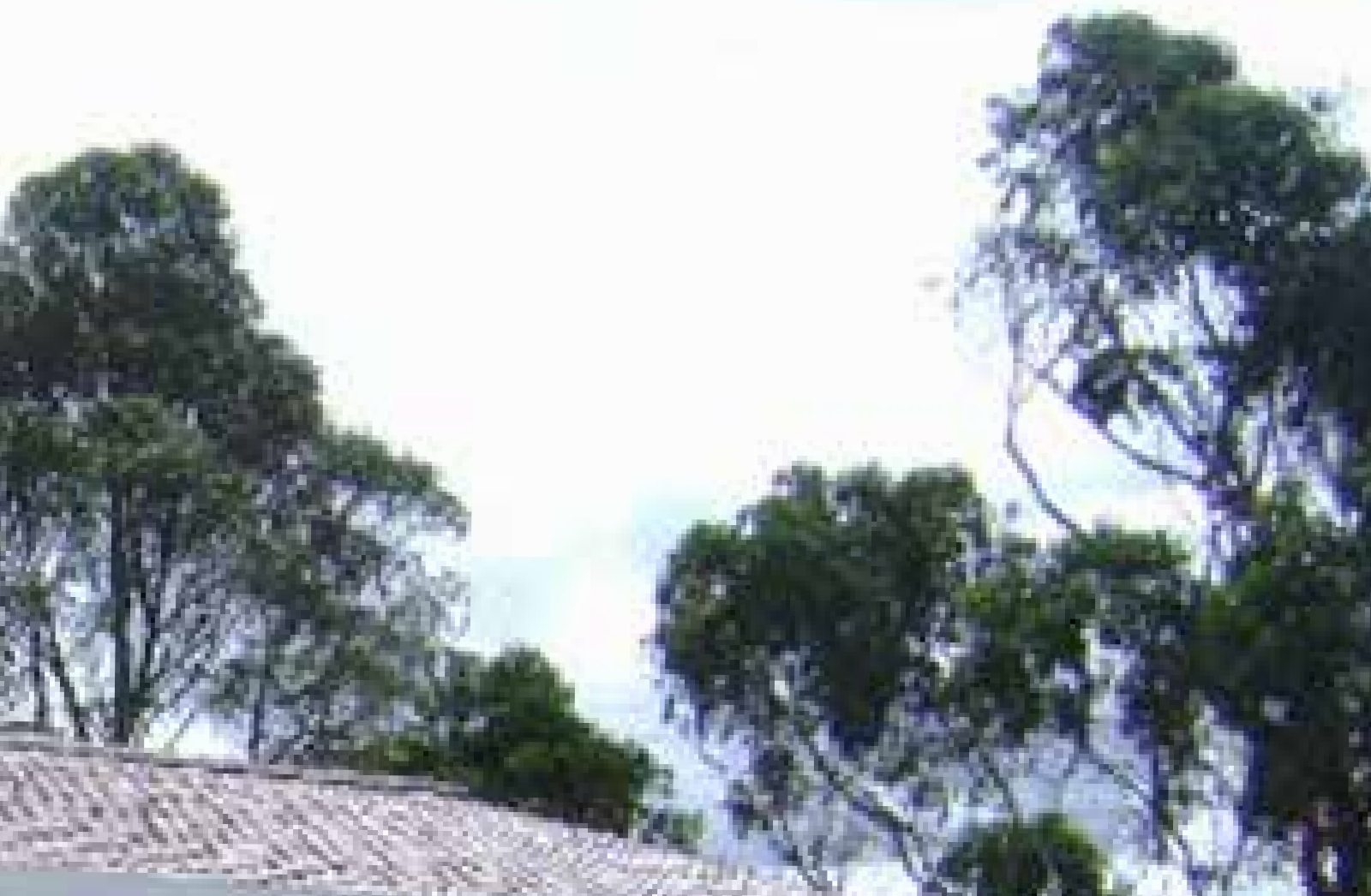

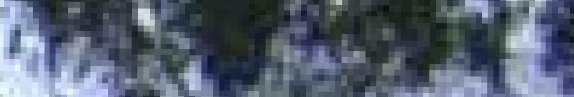

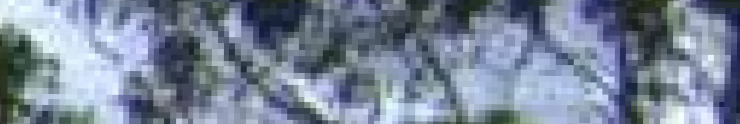
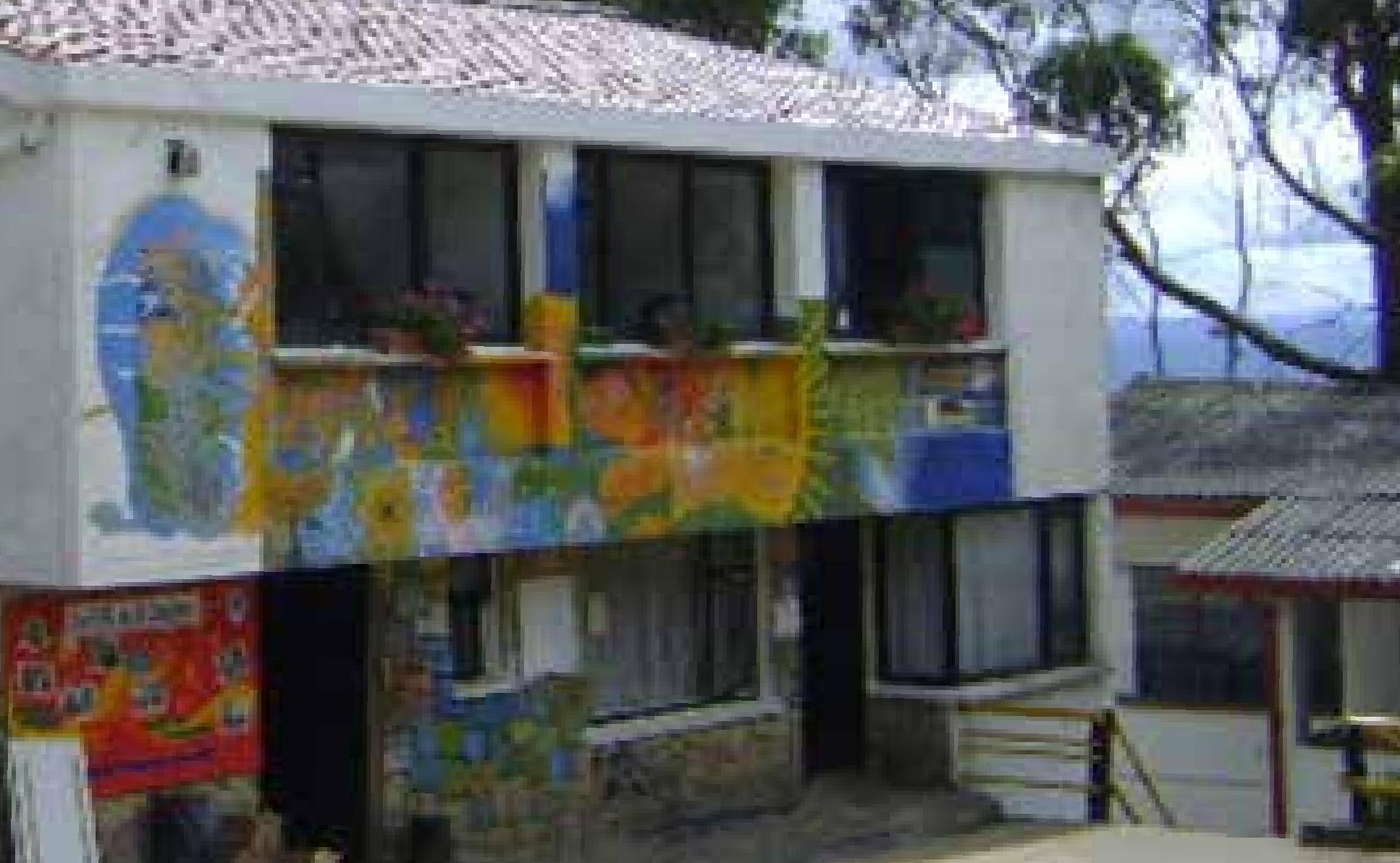

Escuela Pedagógica Experimental. La escuela no debe ser una preparación para la vida; la escuela debe ser la vida misma.

(Elbert Hubard)

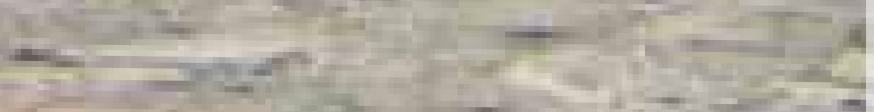

Foto: Autores de articulo 


\section{PRÁCTICA EN LA ESCUELA PEDAGÓGICA EXPERIMENTAL (EPE), UNA AVENTURA POR COMENZAR}

\section{My practicum in the experimental pedagogical school (EPE), an adventure to begin with}

Rosío del Pilar Alarcón Gómez

Fecha de recepción: 27 de febrero de 2013 Fecha de aprobación: 10 de mayo de 2013

\section{Resumen}

El espacio de la práctica desarrollado en la EPE (Escuela Pedagógica Experimental), reconocida alternativa pedagógica, fue toda una aventura. En primera instancia, se hizo una indagación a nivel contextual de la escuela, donde se reconocieron diferentes espacios académicos para ver en qué grupo era más pertinente el trabajo de práctica. Esta dinámica llevó al compromiso con los niveles $5 \mathrm{~A}$ y $9 \mathrm{R}$, que corresponden a tercero y séptimo, respectivamente. Allí, cada nivel desarrolla un proyecto de aula que los estudiantes mismos se plantean para el año; en el nivel cinco, lo que orientó la exploración fueron los sentidos, y en el nivel nueve, la pregunta orientadora fue: ¿Qué pasaría si todas las calles y avenidas de Bogotá se convirtieran en ríos? Este último trabajo permitió el reconocimiento de diferentes sistemas acuáticos; y el abastecimiento de agua por parte de algunos de estos, abriendo paso a trabajos de microhábitats y maquetas de ecosistemas acuáticos, entre otros. Mientras que con el nivel cinco el tiempo de interacción fue menor, abarcando solo algunas prácticas de la exploración de los sentidos.

En medio de este panorama se dio la práctica, que lleva a concluir que lo más importante es cumplirles a los estudiantes lo que se dice o promete; participar en diversas actividades genera ideas y nuevo conocimiento, $y$, finalmente, ser espontáneo y dejar fluir las ideas ante cualquier grupo es lo vital a la hora de abordar la clase, más cuando se trata de trabajo a través de proyectos de aula, donde no se sabe qué puede surgir.

Palabras clave: Práctica pedagógica, proyectos de aula, innovación.

\section{Abstract}

The practice space developed in the EPE (Experimental Pedagogical School), which is recognized for being a pedagogical alternative, was an adventure. First of all, there was a contextual inquiry at the school, which allowed to recognize different academic areas to see which group was more appropriate for the practice work. This dynamic led to commitment with $9 \mathrm{R}$ and $5 \mathrm{~A}$ levels, that corresponding to third and seventh grades, respectively. There, each level develops a classroom project that the own students set ou for the academic year, at level five, which guided the exploration were the senses, and in the level nine, the guiding question was: What would it happen if all streets and avenues in Bogotá became rivers? This latter work allowed the recognition of different aquatic systems, and water supply on their behalf, making works about microhabitats and models of aquatic ecosystems, among others. While the level five interaction time was shorter, covering only some practices of exploration of the senses.

Wthin this outlook this practice took place, leading to the conclusion that the most important is to fulfill what was said or promised to the students, participating in various activities generates new ideas and knowledge, and finally, being spontaneous and let the ideas flow to any group is vital to address the class, especially when it is related to classroom projects, where no one knows what might happen.

Key words: Pedagogical Practicum, Classroom Projects, Innovation.

1 Estudiante de Licenciatura en Biología, primer semestre. Practicante Línea de investigación Desarrollo Sostenible en el área rural. Informe final práctica educativa Correo electrónico: alapirosio@gmail.com 


\section{¿Qué es la EPE?}

"La escuela no debe ser una preparación para la vida; la escuela debe ser la vida misma".

\section{Elbert Hubard}

Inicio con esta frase porque desde hace 35 años, tiempo de constitución de la Escuela Pedagógica Experimental (EPE) - año 1977, ubicada en el kilómetro 4,5 vía La Calera-, allí se promueve construir la felicidad en los estudiantes. Parafraseando al profesor Dino Segura (Director General), no se habla de una felicidad efímera, se busca una felicidad sustentable, donde se forme un complejo de felicidad que recorra la escuela y se extienda por cada una de las familias $y$, a su vez, por toda la sociedad colombiana; a partir de la superación de retos, retos que pueden ser físicos, emocionales o intelectuales, ya que son estas experiencias las que más nos proporcionan felicidad (Segura, 2012).

Así inicié mi exploración, viendo a niños correr y reír por los diferentes espacios de la Escuela, como el bosque, el empedrado y las canchas en la celebración del día del deporte, después de recorrer un camino destapado durante unos cinco minutos por el que me preguntaba: ¿dónde empieza la escuela? ¿Dónde está el celador que me va a preguntar de dónde vengo o a quién necesito? Pero en ninguna de mis visitas apareció, simplemente porque la escuela no tiene puerta, ni celador, es una escuela sin muros y de puertas abiertas.

Esto es parte de la innovación educativa de esta institución, donde la vigilancia y el control no son lo primordial, dejando atrás las posturas tradicionales de control. La EPE fundamenta una transformación en la educación, donde la construcción de una opción de colectivo social diferente contrarreste el ya conformado por una sociedad anclada en valores como la obediencia, la pasividad, la competitividad, el individualismo y la indiferencia (EPE, 2011, p. 7). Todo esto, trabajado a través de tres elementos (confianza, conocimiento y convivencia) que propician un espacio de formación integral, complementaría entre el estudiante, su entorno social y cultural (ibíd.), configurando la Escuela como un eje de transformación cultural de la sociedad.

\section{¿Cómo se organiza la escuela entonces? Una pequeña descripción:}

La escuela está organizada en cuatro ciclos, cada uno con varios niveles (de 0 a 13), todos los estudiantes de la escuela comparten el mismo espacio de recreación. La orientación pedagógica está basada en una concepción de conocimiento desde y para la comprensión de la realidad, donde lo más importante es que las aproximaciones de niños y jóvenes al conocimiento, sean procesos colectivos, de especulación y de vivencias que les permitan construir un mundo y entender la existencia de múltiples realidades (Proyecto Educativo Institucional de la EPE).
Para esto, la metodología que orienta el proceso trata de estrategias pedagógicas ligadas a la consolidación de espacios de formación, tanto en la convivencia como para la construcción de la relación sujeto conocimiento: un ejemplo es el trabajo en torno a proyectos, que pueden ser heterogéneos o de aula. Los proyectos heterogéneos reúnen niños de los diferentes niveles con intereses similares y los proyectos de área se desarrollan desde las inquietudes de los estudiantes canalizadas a través del director de grupo. Fue grato ver cómo se trabaja en cuarto ciclo, los estudiantes pueden elegir una especialización, entre las que se encuentran: teatro, literatura, danza, artesanías o expresión plástica, las cuales les permiten afianzar más en lo que les gusta y concluir su etapa de manera satisfactoria, construyendo obras de teatro, escritos literarios, obras de arte, piezas musicales, objetos personales, entre otros.

En cuanto a la evaluación, es de resaltar que no hay notas ni tareas, pues se busca que, más que tareas corrientes, estas tengan sentido para el aprendizaje del estudiante. Tampoco hay reglamento, ni observador del alumno, como sí se da en una escuela normal; sin embargo, se realizan informes cada dos meses acerca del proceso que llevan los estudiantes, en los que se enfatiza en sus intereses buscando la proyección en la formación. A nivel de convivencia, cada grupo llega a acuerdos con su director de curso y estos son respetados porque son creados por el colectivo. Además, las actividades características de la EPE, como la formulación de proyectos, el desarrollo de eventos y las actividades cotidianas, desde el protagonismo individual y el juego, permiten enfrentar esta etapa generacional por la que atraviesa la escuela, donde los estudiantes cada día son más esquivos a las propuestas y viven pegados a los avances tecnológicos, como iPods, celulares, tabletas, y también a sus problemáticas sociales como noviazgos, drogas, entre otras, que son características de los ciclos 3 y 4 , las cuales son manejadas desde las direcciones de grupo, un espacio más cercano para los estudiantes, dado que no hay orientadora, ni enfermera, y menos psicóloga.

La EPE, se ha construido gracias al tesón de muchos maestros en especial al maestro Dino Segura (fundador - director). Por ello citamos su afirmación con respecto a la formación:

El proceso de formación podría ejemplificarse como la consecución y construcción de las condiciones en que las exploraciones puedan ser libres. Libres en cuanto a los propósitos que se buscan y libres en cuanto a los métodos y estrategias que se utilizan. Podría decirse, de otra manera, que se trata de mantener la actividad en el terreno de lo lúdico, del juego, en el cual no solamente los individuos se la juegan en el proceso, sino que el proceso mismo es un juego (Segura, 2000, p. 36).

Tal vez así se ha construido la EPE. 

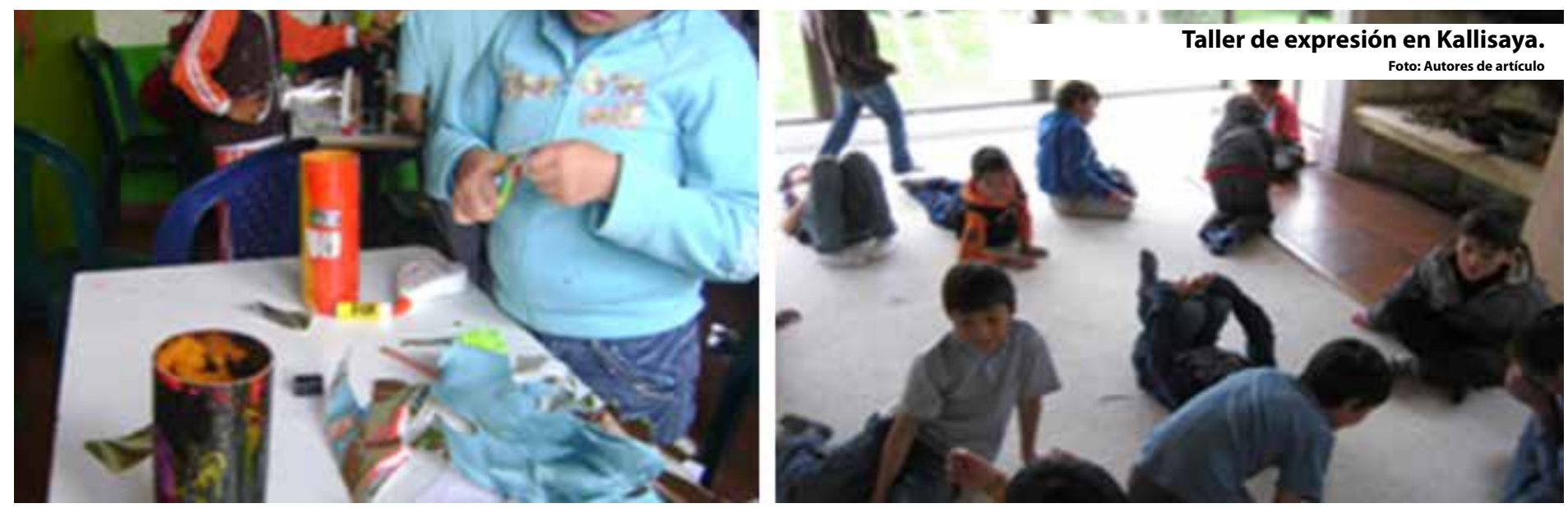

\section{¿Y esta escuela qué tiene que ver con la línea de investigación?}

Como se menciona anteriormente, acerca de posibilitar espacios donde las exploraciones puedan ser libres, tanto en la Escuela como en la Línea de investigación (Enseñanza de la Biología en contextos rurales y urbanos) se da este aspecto. Además la forma de ver la vida desde lo holístico y armónico, consigo mismo y con el ambiente, es uno de los aspectos más interesantes. Aun cuando la EPE es una institución de carácter privado, congrega a estudiantes, maestros y trabajadores en general de diversos lugares de la ciudad, desde Ciudad Bolívar hasta Chía y, por tanto de varios estratos, lo que la hace más diversa, y su ubicación permite la interacción directa con el ambiente, porque se encuentra en los límites de la ciudad, en medio de franjas o sectores de bosque intervenidos por las construcciones en los Cerros orientales, donde también llegan perros como Luisa, Raiza y hasta vacas con sus terneros, que complementan el aprendizaje. Esta comunidad caracterizada por la sencillez y la espontaneidad, permite que se navegue a través de ella y de sus alrededores buscando la integración con la comunidad más cercana para expandir lo que se hace en la escuela y ayudar con las necesidades de la comunidad del sector.

En el caso del proyecto del nivel nueve en el cual se intervino, a partir de las inundaciones sufridas en la ciudad de Bogotá y sus implicaciones se posibilitan múltiples formas del trabajo en el aula, como lo promueve la línea de investigación, iniciando con el reconocimiento del entorno próximo y asumiendo una postura crítica, que será ampliada más adelante.

\section{¿Qué es la práctica?}

Todo lo anterior hace parte de la práctica, definida como:

\begin{abstract}
En el proyecto curricular se concibe la práctica pedagógica como un espacio de reflexión acción e investigación en torno a la innovación, indagación y recontextualización de los saberes desde enfoques éticos, estéticos, cognitivos, pedagógicos y didácticos, en función de la formación profesional e Integral del Maestro en Formación. (Reglamento de Práctica Pedagógica 2012).
\end{abstract}

Al ser la práctica una experiencia y un proceso de auto-construcción y reflexión, digo que esta definición parece muy completa hasta donde se habla de la función, allí solo se tiene en cuenta al practicante, dejando de lado los demás actores de la práctica, se debe pensar en función no solo del proceso integral del maestro en formación, sino en el complemento para la formación integral de todos los participantes que se encuentran en dicha práctica, espacio o momento educativo. Dividida en dos etapas, en el nivel I se hace la consolidación del proyecto de investigación y se desarrolla en el nivel II. Para este caso, tuvo desarrollo desde el primer nivel.

\section{¿Y yo, qué hice allí?}

Estuve durante el primer y segundo semestre de 2012 en la Escuela. Este espacio se logró después de unas deliberaciones que hicieron algunos maestros para la aceptación del trabajo, entendidas como preguntas acerca de lo que iba a hacer en la escuela y el grupo en el que sería más pertinente la práctica; el hecho de no ceñirse a un reglamento no quiere decir que no se tengan acuerdos y una organización previa, así como ciertas exigencias, mas cuando una persona nueva aparece en escena, la selección es conjunta. Así inicié mi aventura por la EPE, llevada de la mano del profesor Arcelio Velasco, quien con muchas expectativas me presenta, y posteriormente me suelta a la toma de decisiones en el campo de manera conjunta con maestros y estudiantes.

Dar una mirada diferente para abordar la Biología fue en principio difícil, pensar en una clase donde no se rayara el tablero era casi imposible, pero a decir verdad en la Escuela lo que menos usé fue el tablero. El hecho de no acogerse a temáticas directamente, sino a problemáticas, hace ver de una forma holística la enseñanza de la biología y, por tanto su aprendizaje al abordar desde diferentes puntos dicha problemática. Veamos cómo me fue.

\section{Primera parte...}

Siguiendo esta dinámica de la escuela tuve la oportunidad de conocer diferentes niveles o cursos, para saber cómo trabajaban en el área de ciencias de acuerdo con el proyecto que estuvieran manejando, y así poder vincularme a uno de los niveles según mis intereses y la pertinencia de la intervención en el grupo. Después de acompañar a los niveles diez, ocho, seis y cinco en algunas clases de ciencias, que vienen siendo los grados octavo, sexto, cuarto y tercero, como se mostró anteriormente, esto gracias al acuerdo dado en la asesoría de ciencias (espacios donde un grupo de maestros discuten semanalmente sobre las prácticas de enseñanza de las ciencias y otras áreas), para que pudiera elegir el grupo después de reconocer los espacios y ver el más adecuado para la práctica, decido enfocar mi experiencia hacia el ni- 
vel cinco y su proyecto de trabajo ¿Qué pasaría si no pudiéramos hablar?, decisión que tomé por varias razones; una de ellas, y tal vez la más importante, el interés de los niños frente al trabajo que realiza el maestro Alex Martínez, licenciado en Literatura con especialización en expresión corporal, ya que siempre se veían muy animados y dinámicos, tanto que se abalanzaban a los maestros apenas los veían.

Los estudiantes del nivel cinco de la EPE se están conociendo (siete -ocho años). Según el desarrollo social por etapas de Erikson (1998), se encuentran en la etapa de industriosidad frente a inferioridad, allí en los niños se comienza a desarrollar una sensación de orgullo en sus logros, inician proyectos y los siguen hasta terminarlos, pero también se puede disgregar la curiosidad y el espíritu investigador si no se enfoca esa energía durante esta etapa.

Este nivel está integrado por 18 estudiantes, en su mayoría niños, solo siete niñas, es muy participativo y unido, aunque se marcan tres grupos: uno el de los niños, todos se unen en la dinámica del juego, se organizan, construyen cuevas, armas y corren en grupo a todo lado (aunque hay un caso especial, y es el de *Felipe, casi nadie lo quiere, todos dicen que es tonto y no juegan con él, los estudios de este caso muestran que él debería estar en un nivel menor, porque con los niños del nivel cuatro se entiende a la perfección), a nivel académico es notorio el liderazgo de otros niños que siempre participan en las clases con aportes muy interesantes y movilizan a sus compañeros.

Las niñas, por su parte, tienen dos grupos; uno, integrado por cinco estudiantes, ellas son muy tiernas y amigables, además de estudiosas, quedan *Julia y *Alejandra, son traviesas, caprichosas, pero también se esfuerzan por realizar sus actividades de la mejor forma, casi todos vienen trabajando desde el primer nivel, por lo que la afectividad y confianza se hacen notar.

Desde la expresión se complementó esta idea, construyendo instrumentos como el trueno, obras de teatro y máscaras. A la vez, se dio la exploración de los sentidos con una mirada artística, científica y divertida, como la visita a la casa taller Kallisaya para fortalecer la enseñanza de las ciencias a partir de la percepción de los sentidos, trabajando desde esta propuesta se construyen múltiples respuestas que muestran la importancia de los órganos de los sentidos, ya que nos permiten relacionarnos con el mundo que nos rodea de manera segura e independiente, también se genera un reconocimiento del cuerpo y de las enfermedades que pueden presentarse y cómo prevenirlas, rescatando que no hay nada que no haya sido aprendido a través de los sentidos; sin embargo, por contar con un tiempo muy corto de acompañamiento en el grupo, no se pudo realizar todo lo planeado.

De manera paralela a esto, se trabaja con el nivel nueve, que es grado séptimo, dividido en dos grupos, el 9R, donde la directora es la profesora Rosa María y el 9G, del profesor Gildardo. La problemática tiene que ver con la importancia del agua, las diferentes afluentes hídricas y ecosistemas acuáticos, se parte de la pregunta ¿qué pasaría si todas las calles de la ciudad de Bogotá se convirtieran en ríos? La tutora Rosa María Galindo, licenciada en Química y quien se encuentra terminando su maestría en enseñanza de las Ciencias, muestra desde su plan de trabajo la importancia del agua como principio de vida que culturalmente los muiscas nos han indicado, y como patrimonio, "un patrimonio que se está acabando, muestra de esta situación es que hoy en el mundo hay 1100 millones de personas que no tienen acceso al agua potable y 2600 millones que carecen de saneamiento básico" (Galindo, 2012). Desde esta panorámica, el curso avanza en el reconocimiento de los diferentes sistemas acuáticos de la ciudad y fuera de ella; es el caso de los lagos, lagunas, represas, aguas termales, ríos, quebradas, humedales, páramos tratando de ubicar estas fuentes en nuestro país. Surgen también otras dinámicas de trabajo como la construcción de microhábitats, que es enfatizada por el nivel 9G, donde se observa el desarrollo de plantas como musgo (Rigodium implexum), lágrima de bebé, para ver la relación del agua con estas, mientras el nivel 9R trabaja sobre cómo sería el 2050 en términos de agua y alimento y la construcción de maquetas de los diferentes ecosistemas.

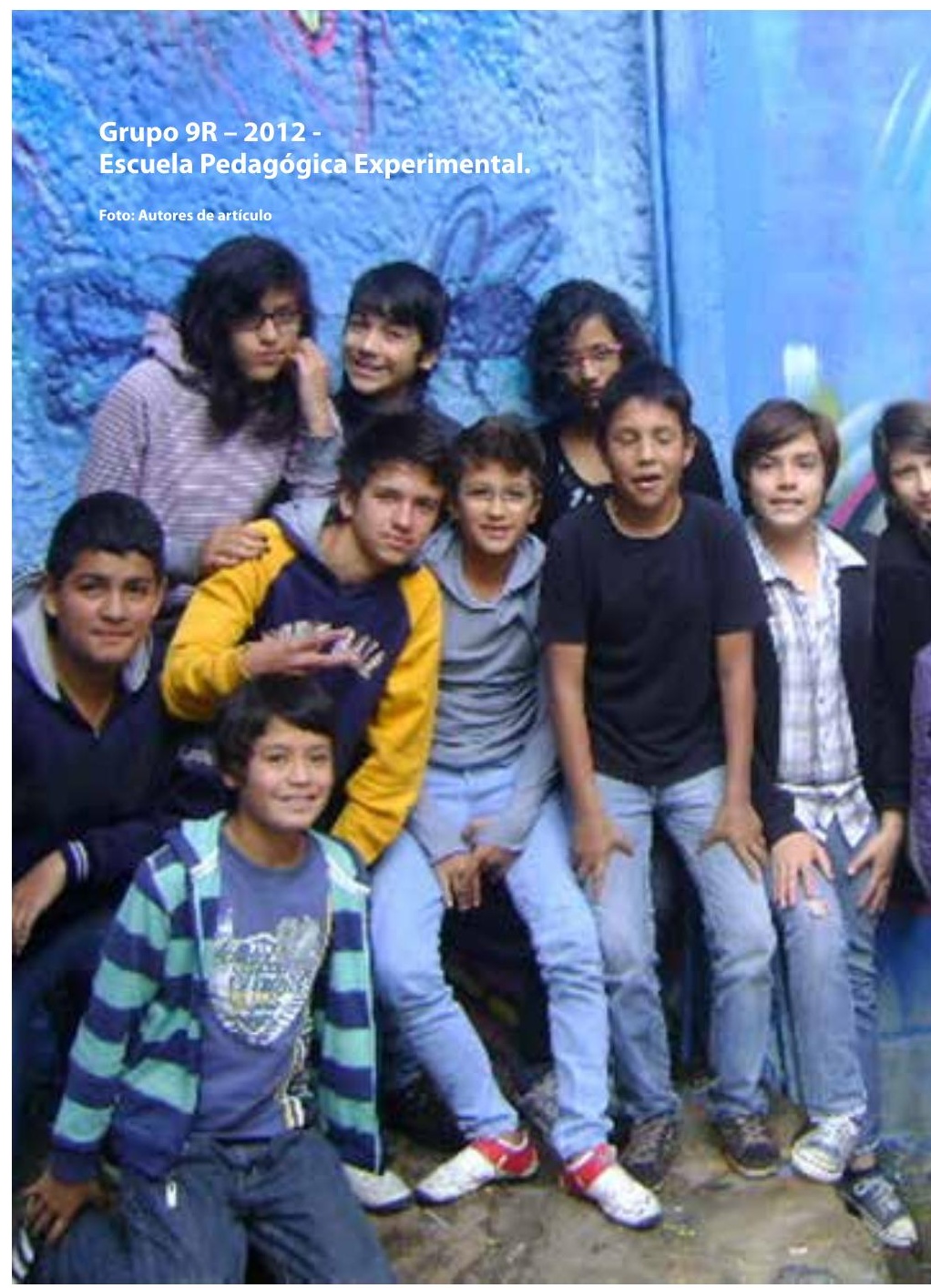


En esta primera etapa, se visita la quebrada Las Delicias, ecosistema alterado que se encuentra en recuperación, nace en los cerros orientales pasa por Chapinero Alto y es parte de la cuenca del río Salitre. A lo largo de la quebrada se están sembrando especies nativas y se realizan jornadas de limpieza, acompañadas de actividades artísticas como la elaboración de murales y eventos publicitarios en la zona, con la participación de artistas como Mauricio Mayorga, Gladys Ortiz y Reinaldo Tibaduiza, pintores y escritores que complementan las actividades que motivan a la comunidad. Esta experiencia da a los estudiantes diferentes puntos de vista para construir "una nueva cultura del agua que transforme las miradas y las acciones de los grupos humanos frente al manejo de este patrimonio" (Guía de trabajo, Galindo 2012).

Todo se complementa con las sesiones de clase que se orientan desde las mismas vivencias, preguntas, reflexiones y el trabajo de cada grupo, como los microhábitats y la problemática del agua a futuro, estas dinámicas son muy participativas. Los grupos, con veinte estudiantes cada uno, son muy diversos pero unidos a la vez (se reúnen en un mismo salón en cambios de clase o espacios libres), los chicos son alegres, activos, dinámicos e inquietos por conocer el mundo que los rodea, su preocupación está centrada en sus relaciones de amistad con sus pares, por la edad en que se encuentran (doce a quince años).

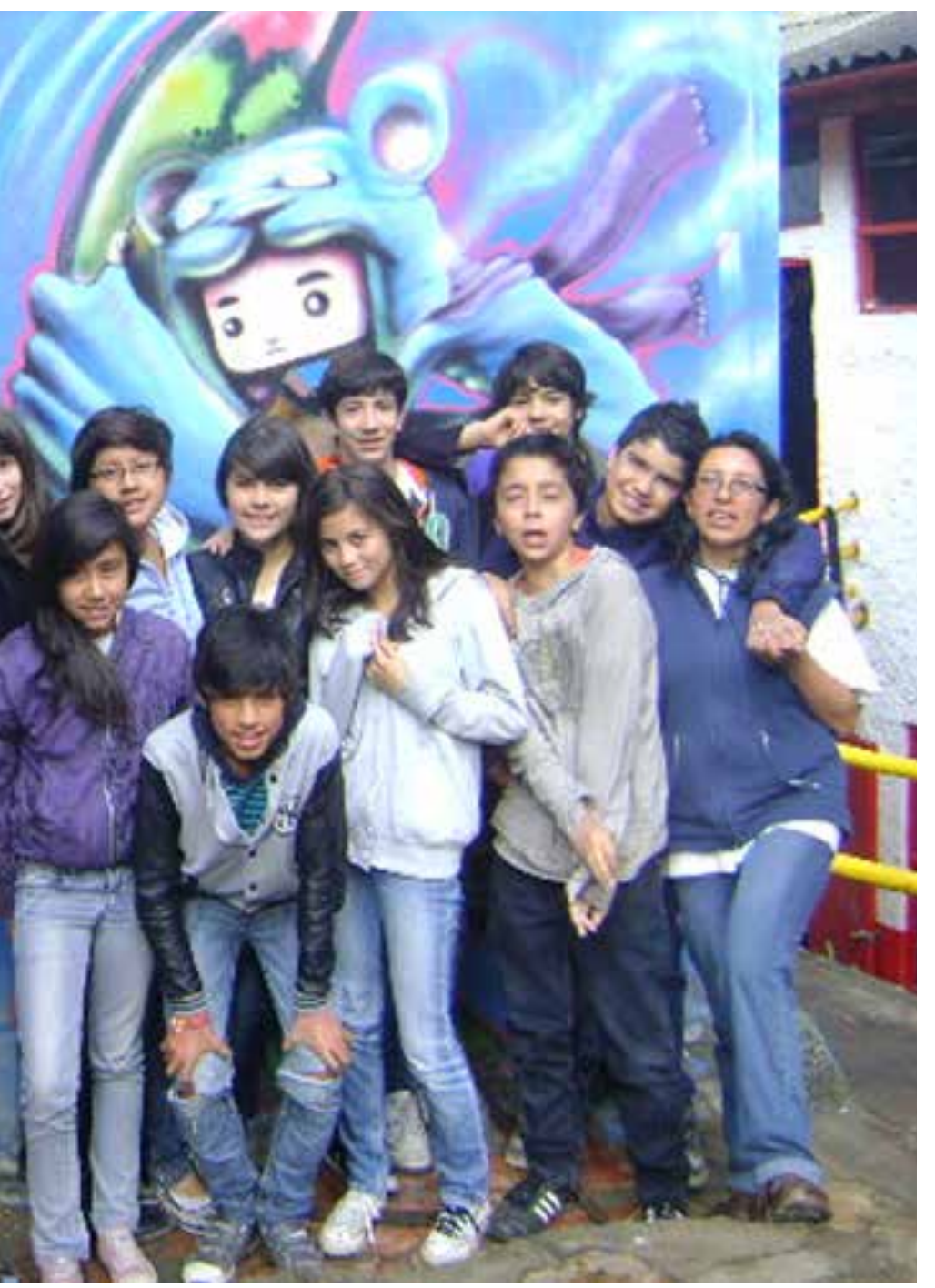

\section{Segunda parte...}

Las salidas pedagógicas continuaron, y estas me enfrentaron más a la realidad que las mismas sesiones en la escuela, era un espacio donde la prioridad no era que me explicaran, sino explicar, explicar algo que no conocía que no había visto. Fuimos a la última vereda de La Calera, Mundo Nuevo, a 2.100 msnm, donde inicia Choachí al sur, a tres horas de la Escuela por carretera destapada; este lugar mágico, tierra de leyendas, con un ecosistema de bosque de niebla, por lo que la llovizna estuvo presente todo el tiempo, nos recibe con la calidad de estas tierras y de su gente, allí se observaban diferentes fuentes hídricas, como las cascadas que son únicas de este lugar, la laguna Brava y el río Blanco, abastecedor del agua de la región; su afluente principal es la quebrada Calostros, que viene del páramo de Chingaza.

A pesar del clima, los estudiantes quedaron encantados con este hermoso lugar, el ejemplo y el carisma de su gente al trabajar en una granja auto sostenible, la lucha por conservar este ecosistema y la voluntad para vivir en un lugar tan aislado y frío, los hizo reflexionar sobre su actuar en la vida cotidiana. Reconocían la importancia de preservar estos lugares en las mejores condiciones, uno de ellos comentaba: "es mágico, parece que no hubiera llegado vida humana a estas tierras" (Alarcón, 2012). En las sesiones de la Escuela, se discutió sobre la importancia de estos ecosistemas en nuestra vida, a lo que los estudiantes respondieron: es vida, abastece de agua a la región, es difícil encontrar un lugar así, la gente lucha mucho para vivir y conservar las condiciones de la vereda, es místico, entre otras.

La última salida que se realizó con el grupo fue al Parque Ecológico Distrital Humedal Santa María del Lago, ubicado en Bogotá entre la avenida Ciudad de Cali y la avenida Boyacá; fue sorprendente para los estudiantes la historia que las guías contaron sobre el humedal, en la que se mostró la reducción en términos de hectáreas que este ha sufrido; en 1936 se contaba con 43 hectáreas y hoy el parque tiene 10,8 hectáreas, así como los espejos de agua, se inició con dos espejos y ahora solo queda uno. Y más aún, es importante saber que es una realidad que se afronta en todo el país.

Se hizo un recorrido de reconocimiento, lo que más les gustó a los estudiantes fue la diversidad vegetal y conocer especies insignias para Bogotá como el Nogal (Juglans regia), participaron en talleres ambientales como Averturphyta, un taller de plantas acuáticas dirigido por estudiantes de la Universidad Pedagógica Nacional, en el que pudieron contrastar los microhábitats de estas plantas con los que ellos trabajan en la escuela, reconocieron varias especies como la lenteja de agua (Lemma minuta), el junco (Juncos effusus) y la enea (Thypa angustifolia), entre otras. Aquí se evidenció el desconocimiento de este tipo de plantas y fue en general una salida muy enriquecedora para ellos, porque tuvieron de cerca el espejo de agua y vieron el ambiente intermedio en el que se encuentran los humedales (tierra agua) y comprendieron más algunas de sus funciones como controlador de inundaciones al actuar como esponja de agua, la importancia a nivel de fertilidad del suelo, y como regulador de la contaminación. Hasta aquí la aproximación de la experiencia durante este año en la Escuela Pedagógica Experimental, 
estos dos proyectos permitieron enriquecer mi formación profesional de manera intelectual, así como a nivel personal, ya que son escenarios diferentes que convergen en la enseñanza de las ciencias biológicas y la importancia de esta para nuestra vida.

\section{¿Qué fue lo más difícil, qué aprendí, qué me re- sultó tranquilo? (Reflexión)}

Esta es quizá la parte más importante de la experiencia a nivel personal, porque afrontar las debilidades, los desaciertos, no es fácil, me gustaría contar algunas particularidades de la práctica respecto a mi desarrollo como maestra en formación, tal vez aquí encuentre la parte más profunda del objetivo de la práctica.

Mi proyecto de investigación didáctica no se trabajó, se transformó de acuerdo con el contexto, porque, como se mencionó, la escuela trabaja por proyectos de Aula, luego ya venían trabajando uno. Inicié reconociendo varios grupos, cada uno con características, directores y formas de trabajo diferentes (tal diversidad me llevó a pensar que con cada grupo se debe tener una forma de trabajo diferente, dependiendo del grupo donde me encontrara), pero finalmente entendí que lo importante es ser espontáneo y dejar fluir las ideas, escuchando primero a los estudiantes y, a partir de ahí enriquecer sus intereses y necesidades personales o académicas de acuerdo con el contexto.

El proceso más difícil fue con los niños del nivel cinco, porque nunca había trabajado con pequeños y, si en algún momento pensé que sería más viable, creo que es un reto mucho más grande del que me imaginé; había que motivarlos todo el tiempo y centrar su atención a través de juegos, lúdicas, chistes, historias sorprendentes o interesantes, algo que poco manejo, les gustaba improvisar todo y estar en actividades jocosas, por esto considero importante trabajar más la expresión corporal y el manejo de grupo a través juegos y rondas, no solo para mí, sino para todos los licenciados.

Otro aspecto que se fue fortaleciendo durante la práctica fue la convivencia tanto con los estudiantes como con los maestros, allí me di cuenta de que lo importante es participar, de lo contrario, difícilmente puede haber algo que una o enriquezca las relaciones y el aprendizaje en grupo, aunque aparte de mis tutores tuve mayor comunicación con las maestras de los ciclos dos y tres, y con el encargado de la huerta, "Don Albertico", un señor que lleva más de diez años trabajando en la Escuela y quien me enseñó mucho sobre plantas empíricamente, muy querido por todos los estudiantes. Entonces, considero que me faltó mayor participación con el resto de maestros de la EPE, y también con los estudiantes, porque, aunque reconozco a muchos, tuve familiaridad con pocos.

Aprendí también que, tanto en la escuela como en la vida, lo importante es ser libre, respetando a los demás, buscando espacios que generen confianza para poder trabajar de manera más tranquila y así poder explotar las potencialidades de cada sujeto, en sí es lograr relacionarse exitosamente con las personas que lo rodean y con el mundo, poder planear, organizar eventos, liderar procesos, aun cuando no se sea hábil en otros aspectos, pero buscando siempre la integralidad. Algo muy importante es que no se deben prometer cosas que no se puedan cumplir, en especial a los niños, porque el otro siempre va a estar esperando aquello que se le dijo, y resulta triste para ellos como deslegitimante para el maestro no cumplir.

Comprendí la importancia de trabajar los temas de forma holística a partir de problemáticas que los mismos estudiantes plantean (lo que se entiende por proyectos de aula), y vi que sí se puede realizar esa exploración a campo abierto articulada con las sesiones de clase, donde esas salidas dejan recuerdos y son una experiencia imborrable, también me gustó la construcción de instrumentos como el trueno, figuras en porcelanicrón y maquetas, en general estuve motivada a las manualidades, cosa a la que antes era esquiva, tal vez porque encontré apoyo desde el tutor, quien daba vía libre, hasta el jefe del taller, quien también se motivaba con estas elaboraciones.

Esto es lo que he podido explorar en esta oportunidad, que se aprovechó de la mejor manera. El compromiso de la EPE, acompañado de la confianza, haciendo innecesarias las reglas externas, y posibilitando la auto organización, permitió una experiencia mucho más provechosa y tranquila.

\section{Otra mirada...}

Como complemento, con la participación en diferentes espacios y escenarios de la escuela que muestran cómo se construye conocimiento (jornadas deportivas, día del edicto, obras de teatro, visita de universidades, conferencias sobre economía azul, entre otras) se puede dar un acercamiento de los estudiantes y el proceso que llevan, del que a nivel general puedo decir que cada uno de los estudiantes se desarrolla desde sus intereses, la flexibilidad y oportunidad que tiene para ello en la escuela; sin embargo, falta autonomía y responsabilidad por parte de algunos para vivir la escuela, ya que unos duran mucho tiempo en entender el compromiso y lograr la autonomía, otros llevan poco tiempo en la Escuela y aún se ven desubicados, además, los jóvenes atraviesan una generación muy particular, para la que sus intereses van ligados a la adrenalina o las emociones que puedan experimentar en las diferentes actividades, bien sea el deporte, el estudio, las drogas o compartir con sus compañeros (tomado de seminarios con los maestros de la escuela y evidenciado personalmente). Esto, dado que la innovación genera nuevas ideas, pero también pueden aparecer situaciones imprevistas. Por eso la aseveración del director Dino Segura: "El tipo de relaciones sociales que se están estableciendo en nuestros días conducen a la formación y deformación de nuestros niños en sus valores y su carácter".

\section{Agradecimientos}

A la línea de investigación Enseñanza de la biología en contextos rurales y urbanos, por brindar estos espacios y al profesor Arcelio Velasco por promover mi práctica en esta institución, su acompañamiento y apoyo durante esta. A la $E P E$, por abrirme el espacio y poder elegir con qué niveles trabajar. A mi tutora, Rosa María Galindo, por ser un ejemplo como persona y como maestra incansable, que no deja 
de soñar. Al profesor Alexander Martínez, por su paciencia y gran virtud para trabajar con los pequeños. A todos los estudiantes y personal de la Escuela, con quienes compartí y me enseñaron muchas cosas, en especial a mis compañeros de ruta, los tocayos Gabriel, a "Don Albertico", Óscar y las maestras Alicia, Solita, Deyanira, Patricia Fernández y al profesor Jorge, mil gracias a todos.

Porque todas las actividades deben ser una excusa para caminar juntos por el mundo, este poema, dedicado por una estudiante a la Escuela en la celebración de sus 35 años (primero y 2 de noviembre de 2012), resume lo que la escuela se propone:

\section{LA ESCUELA DE LOS NIÑOS FELICES}

Te voy a confiar un secreto:

Hay una escuela donde no se aprende a deletrear, sino a cabalgar sobre ciervos.

Tampoco se aprende a mirar fijamente a la pizarra con ojos soñolientos, sino a navegar sobre nubes.

No a medir las carreras con cronómetro, ni los saltos con cinta métrica, sino a bailar sobre el alambre.

No se aprende a bajar la cabeza, ni a mirar de reojo al maestro, sino a domar monstruos.

Tampoco a balbucear textos, sino a reconocer huellas de hadas.

Y nada de que dos y dos son cuatro, y la hora tiene sesenta minutos, sino a hacer magia y a soñar.

No a estar sentado en las bellas mañanas de primavera en un aula que huele a trapo de pizarra y a ropa sudada, sino a oler como las flores.

No a pedir buenas notas y a temblar cuando van a ser entregadas, sino a caminar sobre el agua.

Allí tampoco se aprende que la luna empieza con "I" estrella se escribe con "II" y que lobo tiene una "b" sino a hablar el lenguaje de los animales.

No a estar sentado inmóvil y con la boca cerrada, sino a vivir en los árboles.

Y mucho menos a empujar a los demás:

"iLargo! Yo primero".

sino a consolar a las personas tristes.

¿Qué dónde está esa escuela?

En el kilometro 4,5 vía La Calera. Se llama "La Escuela Pedagógica Experimental, la escuela de los Niños Felices".

Su puerta está abierta de par en par. Vete allí.

Y si un día regresas, cuéntales a tus maestros dónde estuviste.

Quizá comiencen a escucharte.

\section{Referencias bibliográficas}

Alarcón, R. (2012). Notas de la práctica. Grabaciones. Manuscrito no publicado. Universidad Pedagógica Nacional. Bogotá. Colombia.

Brunner, J. (1988). Realidad mental y mundos posibles. Los actos de la imaginación que dan sentido a la experiencia. Barcelona: Gedisa.

Escuela Pedagógica Experimental. (2011a). Proyecto Educativo Institucional (PEI). ¿Cómo son las búsquedas pedagógicas? Proyectos de Aula. Recuperado de: http://www.epe.edu.co/Proyecto-Educativo-Institucional?artpage $=8-9$

Escuela Pedagógica Experimental. (2011b). El ambiente educativo. Recuperado de: http://www.epe.edu.co/

Erikson, E. (1998). Los Ocho Estadios del Desarrollo Psicosocial. Recuperado de: http://es.scribd.com/doc/63716120/8-Etapas-de-Erikson-Del-Desarrollo-Psicosocial

Flórez, R. (1999). Evaluación Pedagógica y cognición. Bogotá: Editorial Mc Graw - Hill.

Gómez, M. Lizarralde, M. y Segura, D. (2008). Conviviry aprender. Hacia una escuela alternativa. Bogotá: Colección Polémica Educativa.

Galindo, R. (2012). Plan de trabajo nivel nueve R, ¿Qué pasaría si las calles y avenidas de Bogotá se convirtieran en ríos? Escuela Pedagógica Experimental. Bogotá. Colombia.

Richardson, K. (1998). Modelos de desarrollo cognitivo. Madrid: Editorial Alianza.

Segura, D. (1999). La construcción de la confianza: una experiencia en proyectos de aula. Bogotá (Colombia): Colección Polémica Educativa.

Segura, D. (2000). ¿Es posible pensar otra escuela? Bogotá.

Segura, D. (2008). Las Urgencias de la Innovación. Documento presentado en Costa Rica en el evento $10^{\circ}$ Congreso Nacional de Ciencias y Estudios Sociales Recuperado de: www.scribd.com/doc/8093805/Dino-Segura

Segura, D. et al. (1999). Constructivismo. ¿Construir qué? Bogotá (Colombia): Colección Polémica Educativa. 